\title{
Application de la charte de l'éthique des facultés de médecine : définition des premières orientations stratégiques
}

\author{
Implementation of the Chart of Ethics for Medical Faculties: \\ definition of first strategic orientations.
}

Lucie BRAZEAU-LAMONTAGNE ${ }^{1}$, Jacques Henri BARRIER ${ }^{2}$, Somian Francis EHUA ${ }^{3}$

\begin{abstract}
Résumé Contexte : Dès sa publication, il est apparu que la charte de l'éthique des facultés de médecine (CEFM) devait être accompagnée d'orientations stratégiques pour connaître une application effective. Méthode: À Cotonou, en avril 2005, à l'occasion des Journées universitaires francophones de pédagogie médicale (JUFPM), un atelier stratégique (AS) a été dédié à l'élaboration de telles orientations stratégiques. Cette approche méthodologique a déjà permis de créer le comité international de la CEFM. Résultats : Les orientations stratégiques concernent les obstacles anticipés à l'application de la CEFM, les stratégies perçues comme étant prioritaires pour l'application de la CEFM et les indicateurs pressentis pour suivre les applications de la CEFM. Discussion : Pour que la CEFM soit appliquée, il est nécessaire : que se développent des espaces de communication et d'expression dans ce domaine; que chaque faculté affiche et démontre son engagement institutionnel envers les personnes qui la constituent et envers les institutions avec qui elle est affiliée, tant pour ses programmes de formation que pour ses programmes de recherche; que soient identifiés des indicateurs de cet engagement dans chaque faculté et entre les facultés. Enfin il est apparu essentiel que la Conférence internationale des doyens et des facultés de médecine d'expression française (CIDMEF) tienne ellemême explicitement compte de la CEFM dans tous ses travaux et dans les soutiens qu'elle apporte aux facultés membres de la CIDMEF Conclusion : La CEFM représente un engagement institutionnel qui a besoin d'alimenter ses orientations stratégiques par le débat sur ses applications et le partage des fruits de ses applications. Les idées issues de cet AS ont conduit la CIDMEF à la création de l'Observatoire de la CEFM.
\end{abstract}

Mots clés Ethique ; éducation médicale ; faculté de médecine ; charte.

Abstract Context: From its very beginning, it was obvious that the Chart of Ethics for Medical Faculties (CEMF) needed practical strategic orientations to reach genuine effectiveness. Method: During the "Journées universitaires francophones de pédagogie medicale" (JUFPM) meeting held in Cotonou in April 2005, a strategic workshop (SW) has broached the first practical strategic orientations for the CEMF application. SW follows the approach that created the CEMF project itself. Results: Strategic orientations were aimed to: anticipated hurdles for the CEFM implementation, strategies perceived as priority for the CEFM application and accessible indicators to allow the CEFM implementation follow up. Discussion: For the CEMF occurrence, it firstly must create discussion opportunities in this field. Moreover, it needs evidence of institutional commitment from personal as well as from formation/research programs in each affiliated Medical Faculties. It also requires indicators for institutional commitment within each Faculty and between them. It finally requests the participation of the CIDMEF ("Conférence internationale des doyens et des facultés de médecine d'expression française") to include the CEMF in its working activities. Conclusion: The CEMF represents an institutional endowment that needs to be nurtured with debates on orientations and to share its various applications. From what was elucidated by the SW in Cotonou, the "Observatoire de la CEFM" stands out as a practical and strategic step.

Key words Ethics; medical education; medical school.

Pédagogie Médicale 2006;7:59-62

1- Secrétaire du Comité de la charte de l'éthique des facultés de médecine (CCEFM) - Sherbrooke (Québec, Canada)

2- Président du Conseil Pédagogique de la CIDMEF - Coordonnateur du CCEFM - Nantes (France)

3- Membre du CCEFM - Abidjan (Côte d'Ivoire)

Correspondance : Lucie Brazeau-Lamontagne - Département de Radiologie diagnostique - Centre hospitalier et Faculté de Médecine de l'Université de Sherbrooke - 3001, 12 e avenue Nord - Sherbrooke, Qc - Canada J1H 5N4 - Tél. (819) 346-1110 poste 14985 - Télécopieur : (819) 820-6423 - Mailto:Lucie.Brazeau@USherbrooke.ca 


\section{Introduction}

La Charte de l'éthique des facultés de médecine (CEFM) ${ }^{1}$ a été adoptée le 14 mai 2004 par le Bureau permanent de la Conférence internationale des doyens et des facultés de médecine d'expression française (CIDMEF). Un mois plus tard, elle a été présentée à Sherbrooke au cours du troisième Forum international francophone de pédagogie médicale. À cette occasion, il est apparu évident qu'une réflexion sur les applications prévisibles de la CEFM s'imposait et ce, rapidement. C'est dans ce sens que l'atelier stratégique (AS) sur les applications de la CEFM a été conçu ; il a été proposé dès les Journées universitaires francophones de pédagogie médicale (JUFPM) organisées à Cotonou en 2005. L'objectif de l'AS ${ }^{2}$ était de dresser, en s'appuyant sur les intervenants des milieux académiques que regroupe la CIDMEF, un inventaire des applications de CEFM à mettre en oeuvre dans les facultés de médecine. Les facultés de médecine visées sont celles qui sont membres de la CIDMEF mais sans exclure les autres.

\section{Méthode}

La méthode de l'atelier stratégique (AS) a déjà conduit à la création du comité international de la CEFM. L'AS a été élaboré à partir des idées qui ressortaient du Forum international francophone de pédagogie médicale de Sherbrooke de 2004. Accepté par le comité scientifique des XVI Is JUFPM, il s'est tenu à Cotonou le 7 avril 2005. Au total 21 personnes, issues des continents africain, américain et européen ont été impliquées dans cet atelier qui s'est déroulé pendant trois heures de la manière suivante. Après une introduction sur l'origine et la définition de la CEFM, les participants ont débattu des trois questions suivantes : 1) Quelles sont les principales difficultés d'application de la CEFM ? 2) Quelles sont les deux ou trois actions stratégiques à envisager ? 3) Comment suivre la mise en application de la CEFM ?

Les participants (18) étaient tous impliqués dans les milieux de formation médicale et de sciences de la santé. Ils ont été répartis en deux groupes de neuf participants chacun. La distribution entre les deux groupes s'est faite de manière spontanée, selon la commodité des lieux. Après 20 minutes de discussion pour chacune des questions, une petite discussion plénière de 20 minutes a permis de dégager les idées fortes communes aux deux groupes de discussion, tout en laissant émerger les idées nouvelles que cette mise en commun suscitait à l'égard de chacune des trois questions. Les deux animateurs sont demeurés disponibles pour les questions de clarification durant les discussions en petits groupes puis ils sont intervenus durant les séances plénières de la manière suivante. La rédaction des idées fortes s'est faite séance tenante, c'est-à-dire durant la discussion plénière dédiée à chaque question; les idées fortes étaient projetées sur écran à mesure qu'elles étaient écrites sur ordinateur par l'un des animateurs pendant que l'autre animait le groupe plénier. Cela a permis de valider au fur et à mesure, question par question, la synthèse des propos échangés sous forme d'idées fortes consensuelles. Durant la dernière période de l'AS, les idées fortes ont été à nouveau projetées devant tout le groupe. Cette dernière étape a permis les corrections finales en assurant une seconde validation, en vue de rapporter officiellement les résultats de l'AS. Cette version finale validée constitue les résultats qui suivent.

\section{Résultats}

Les résultats présentés ici reproduisent les énoncés validés durant l'AS en réponse aux trois questions posées.

Question 1. La CEFM s'adresse à plusieurs secteurs. Vous êtes responsable de la mettre en application dans votre faculté. Quelles sont les deux ou trois principales difficultés que vous anticipez pouvant faire échec à cette mise en application?

\section{Synthèse des idées fortes:}

- Il n'y a pas de constat du besoin de la CEFM. Or, on ne peut pas adhérer à une charte sans en expliquer la motivation.

- L'éthique est un sujet surclassé par les difficultés de moyens.

- Le milieu des facultés de médecine est sous une forte influence divergente venant de la marchandisation de la médecine.

- Le contexte où baignent les facultés de médecine montre de nombreuses manifestations d'inégalité / inéquité dans l'accès aux soins.

- La CEFM ne fournit ni ne doit fournir son mode d'application dans son texte même car les applications doivent puiser leur pertinence dans les contextes particuliers. Les contextes particuliers ne sont pas universels, par définition. - Il n'existe pas de document sur les avenues facilitant la mise en application de la CEFM. Faute de pistes éclairant ses mises en application, la CEFM demeurera lettre morte, perdue dans le hiatus entre les voeux pieux et la réalité.

- Les facultés de médecine sont soumises à une forte influence/ingérence extérieure (tutelles ministérielles, dépendance envers les milieux hospitaliers, etc.). Par conséquent, même si elles souhaitent mettre la CEFM en 
application, la latitude décisionnelle des facultés est limitée, voire freinée.

- La mise en application de la CEFM dépend de la coopération entre les facultés de médecine ; cette coopération dépend à son tour de la solidarité entre les facultés de médecine qui doit s'exprimer en gestes de réciprocité. Or, il existe actuellement une culture de compétition entre les facultés de médecine, peu propice à la coopération.

- Comment adapter une charte sans risque de la dénaturer ? D'où l'importance de dégager des pistes de mise en application plutôt que de viser une adaptation « continent par continent ».

- Les doyens ne se sont pas approprié la CEFM : "J’ai déjà un plan stratégique pour ma Faculté! Quoi encore?"

- La question préalable de l'évaluation de conformité à la CEFM n'est pas éclaircie.

- La résistance des professeurs à l'émergence des questions touchant l'éthique ; ils sont inquiets d'ouvrir "sur leur jardin intérieur ».

Question 2. En qualité de RESPONSABLE de la mise en application de la CEFM dans votre faculté, quelles sont les deux ou trois actions les plus stratégiques que vous choisissez D'ABORD?

Synthèse des idées fortes:

- Créer un comité/conseil de l'éthique où la représentativité minimale professeurs/étudiants/société civile/praticiens généralistes serait assurée et à qui serait confiée la sensibilisation des acteurs dans la faculté.

- Inventorier des urgences éthiques selon le contexte du pays : enquêtes des manquements dans l'environnement de la faculté en vue de cibler les points cruciaux pour la formation à mettre en place ; émergence des besoins ressentis dans le milieu de manière à y répondre.

- Intégrer l'enseignement de l'éthique dès la séance « inaugurale » et y présenter la CEFM.

- Favoriser l'adhésion/appropriation de la CEFM par l'organisation de séminaires/ateliers regroupant les professeurs, les comités nationaux d'éthique.

- Soutenir la crédibilité du doyen par l'équité des critères de sélections pour tous les groupes à la faculté.

- Fournir les moyens et instruments pédagogiques au renouveau éthique dans la faculté.

- Définir un plan d'action de formation à l'enseignement de l'éthique à partir d'une enquête connaissance/ attitude/pratique.

Question 3. En qualité de RESPONSABLE de la mise en application de la CEFM dans votre faculté, quels indicateurs choisissez-vous pour évaluer sa mise en

\section{application EFFECTIVE et quel rôle envisagez-vous que la CIDMEF doive y jouer?}

\section{Synthèse des idées fortes:}

Concernant l'application effective de la CEFM :

- Identifier la structure responsable de la mise en application de la CEFM dans la faculté.

- Retracer les textes expliquant la pertinence et les applications particulières de la CEFM à la faculté.

- Recueillir le témoignage des étudiants et des enseignants sur la visibilité de la CEFM dans la faculté et sur ses effets identifiables.

- Rechercher l'évidence d'un mécanisme/procédure permettant l'adhésion personnelle des " acteurs » à la CEFM au sein d'une faculté.

- Suivre l'élaboration d'un plan stratégique pour mettre en application la CEFM

Concernant les rôles de la CIDMEF :

- Étudier/suivre l'adhésion à la CEFM selon les réalités socio-culturelles et économiques de chaque pays.

- Fournir des exemples des "bonnes pratiques " dont la CIDMEF doit transmettre le témoignage aux différentes tutelles des facultés.

- Inclure la mise en application effective de la CEFM dans les critères de la procédure d'évaluation institutionnelle des facultés de médecine faite par la CIDMEF.

- Aider à l'élaboration des indicateurs d'application effective (pour une faculté, pour un programme de formation, pour un programme de recherche, etc.), avec une attention particulière pour inclure des indicateurs aussi bien qualitatifs que quantitatifs.

- Aider au développement d'outils pédagogiques adaptés à l'apprentissage de l'éthique dans les facultés de médecine, programme de formation par programme de formation.

\section{Discussion}

Cette discussion est le fruit du travail d'analyse que les responsables de cet AS ont fait des idées fortes après l'AS; elle n'a pas été élaborée durant l'AS.

Selon les responsables de cet AS, des orientations stratégiques peuvent être déduites des idées fortes émises lors de l'AS. D'abord, l'espace permettant un débat large des applications de la CEFM fait défaut. Si les moyens concrets pertinents et adaptés pour mettre la CEFM en application sont à définir, l'intérêt que ces moyens suscitent peut rassembler de manière productive des intervenants enseignants issus de différents continents. Enfin l'aide mutuelle que les facultés de médecine peuvent 


\section{Communication brève}

s'apporter dans la perspective de la CEFM souffre du manque d'outils adaptés. Néanmoins cette aide, y compris celle de la CIDMEF, parait non seulement pertinente mais nécessaire pour arriver à mettre vraiment la CEFM en application. Plus localement, dans une faculté précise, l'adhésion des individus à la CEFM, bien que nécessaire, ne peut pas porter ses fruits sans que l'engagement de chacun soit connu et partagé par la communauté institutionnelle en tant que collectivité vivante. Faute de cette appropriation institutionnelle authentique, les moyens et les stratégies d'application de la CEFM demeureront lettre morte. Ces moyens incluent le développement des outils pédagogiques adaptés à l'apprentissage de l'éthique professionnelle, assortis de leurs outils d'évaluation. L'autorité morale de la CIDMEF paraît pouvoir influencer les tutelles des facultés, d'autant mieux qu'elle donnera elle-même l'exemple en rendant accessibles ses travaux qu'elle maintiendra explicitement cohérents avec la CEFM.

\section{Conclusion/Développement}

Cette synthèse a abouti à l'idée d'observatoire de la CEFM. Cet observatoire prend la forme d'un site internet (blogue). Il se propose de suivre les effets de la CEFM, d'en stimuler les références et les applications. Il est aussi une médiathèque (références et hyperliens), doublée d'un forum servant de plate-forme virtuelle à des partenaires, tous reliés, à des niveaux différents, par la démarche de la CEFM. Il deviendra au fil de sa construction une source d'informations et d'échanges à propos de la CEFM et de ses applications. Ce blogue est déjà accessible en ligne : www.sante.univ-nantes.fr/med/ethique. Il est conçu pour connaître une amélioration constante, comme la CEFM elle-même.

\section{Contributions}

Lucie Brazeau-Lamontagne, Jacques Henri Barrier et Francis Somian Ehua ont élaboré l'atelier stratégique (AS); Lucie Brazeau-Lamontagne et Francis Somian Ehua ont animé l'AS ; Lucie Brazeau-Lamontagne et Jacques Henri Barrier ont fait la synthèse des résultats obtenus ; Lucie Brazeau-Lamontagne a rédigé l'article ; Jacques Henri Barrier et Francis Somian Ehua ont révisé le manuscrit.

\section{Références}

1. Conférence internationale des doyens et des facultés de médecine d'expression française (CIDMEF). Charte de l'éthique des facultés de médecine. Pédagogie Médicale 2004;5:211-7.

2. Brazeau-Lamontagne L, Ehua SF. Application de la charte de l'ethique de la CIDMEF XVIe Journées Universitaires Francophones de Pédagogie Médicale de la Conférence Internationale des Doyens et des Facultés de Médecine d'Expression Française, Cotonou (République du Bénin), 5-8 avril 2005. In : Conférence internationale des doyens et des facultés de médecine d'expression française (CIDMEF). Programme et volume des communications, 2005:3.

3. Barrier JH, Brazeau-Lamontagne L. La charte de l'éthique des facultés de médecine : un cadre conceptuel et un outil. Pédagogie Médicale 2004;5:197-9.

Manuscrit reçu le 2 novembre 2005 ; commentaires éditoriaux formulés aux auteurs le 4 novembre 2005 et le 4 janvier 2006; accepté pour publication le 6 janvier 2006. 